\title{
HIF-2a-induced chemokines stimulate motility of fibroblast-like synoviocytes and chondrocytes into the cartilage-pannus interface in experimental rheumatoid arthritis mouse models
}

Yun Hyun Huh ${ }^{1 \dagger}$, Gyuseok Lee ${ }^{2 \dagger}$, Keun-Bae Lee ${ }^{3}$, Jeong-Tae Koh ${ }^{4,5}$, Jang-Soo Chun ${ }^{2 *}$ and Je-Hwang Ryu ${ }^{4,5^{*}}$

\begin{abstract}
Introduction: Pannus formation and resulting cartilage destruction during rheumatoid arthritis (RA) depends on the migration of synoviocytes to cartilage tissue. Here, we focused on the role of hypoxia-inducible factor (HIF)-2a-induced chemokines by chondrocytes in the regulation of fibroblast-like synoviocyte (FLS) migration into the cartilage-pannus interface and cartilage erosion.

Methods: Collagen-induced arthritis (CIA), K/BxN serum transfer, and tumor necrosis factor-a transgenic mice were used as experimental RA models. Expression patterns of HIF-2a and chemokines were determined via immunostaining, Western blotting and RT-PCR. FLS motility was evaluated using transwell migration and invasion assays. The specific role of HIF-2a was determined via local deletion of HIF-2a in joint tissues or using conditional knockout (KO) mice. Cartilage destruction, synovitis and pannus formation were assessed via histological analysis.

Results: HIF-2a and various chemokines were markedly upregulated in degenerating cartilage and pannus of RA joints. HIF-2a induced chemokine expression by chondrocytes in both primary culture and cartilage tissue. HIF-2a -induced chemokines by chondrocytes regulated the migration and invasion of FLS. Local deletion of HIF-2a in joint tissues inhibited pannus formation adjacent to cartilage tissue and cartilage destruction caused by K/BxN serum transfer. Furthermore, conditional knockout of HIF-2a in cartilage blocked pannus formation in adjacent cartilage but not bone tissue, along with inhibition of cartilage erosion caused by $\mathrm{K} / \mathrm{BxN}$ serum transfer.
\end{abstract}

Conclusion: Our findings suggest that chemokines induced by IL-1 $\beta$ or HIF-2 $\alpha$ in chondrocytes regulate pannus expansion by stimulating FLS migration and invasion, leading to cartilage erosion during RA pathogenesis.

Keywords: Rheumatoid arthritis, Cartilage destruction, Chemokine, HIF-2a

\section{Introduction}

Rheumatoid arthritis (RA) is a chronic autoimmune disease causing synovial inflammation, bone erosion, and cartilage destruction. The inflammatory process initially affects a single joint, but the disease usually progresses to affect nearly

\footnotetext{
* Correspondence: jschun@gist.ac.kr; jesryu@jnu.ac.kr

${ }^{\dagger}$ Equal contributors

${ }^{2}$ Cell Dynamics Research Center and School of Life Science, Gwangju Institute of Science and Technology, 123 Cheomdangwagi-ro, Buk-gu, Gwangju 61005, Republic of Korea

${ }^{4}$ Department of Pharmacology and Dental Therapeutics, School of Dentistry, Chonnam National University, 77 Yongbong-ro, Buk-gu, Gwangju 61186, Republic of Korea

Full list of author information is available at the end of the article
}

all joints [1]. RA is characterized by abnormal synovial hyperplasia caused by increased proliferation and activation of synoviocytes in inflamed synovia [2,3]. The pannus, an aggressive front of hyperplastic synovium, invades and destroys mineralized cartilage and bone [4-6]. Migration and invasion of activated fibroblast-like synoviocytes (FLS) into cartilage and bone are critical events during invasive pannus formation in RA synovium [4-6]. However, little is currently known on the molecular mechanisms underlying migration and invasion of activated FLS and formation of pannus.

FLS in the inflamed synovium produce numerous catabolic regulators, including chemokines, cytokines, and matrix metalloproteases (MMPs), which are directly 
implicated in joint destruction [7, 8]. Production of these factors, in addition to cell-cell interactions, is crucial for progression of RA $[8,9]$. For instance, FLS produce $\mathrm{CX}_{3} \mathrm{CL} 1$, which attracts $\mathrm{T}$ cells to the joint [10]. T cells promote tumor necrosis factor alpha (TNFo) production, in turn causing FLS proliferation [10]. FLS in inflamed synovium also produce several other chemokines, including CXCL8, CXCL9, CXCL10, CXCL12, CCL2, and CCL3 [7, 11-13]. Chemokines are key players in inducing directed chemotaxis in nearby responsive cells and are classified into four subfamilies, specifically $\mathrm{CXC}, \mathrm{CC}, \mathrm{CX}_{3} \mathrm{C}$, and XC [14]. These signaling proteins are essential for leukocyte trafficking during chronic inflammatory diseases, such as RA, atherosclerosis, and adipose inflammation [15], and additionally stimulate migration, proliferation, and MMP activation in FLS during RA pathogenesis [16]. Chemokines are also produced by chondrocytes in cartilage tissues of RA joints [17-19], but the potential role of hypoxia-inducible factor (HIF)-2 $\alpha$-induced chemokines in regulation of RA pathogenesis remains to be explored.

Previously, we demonstrated that HIF- $2 \alpha$ (encoded by Epas 1), a transcription factor regulated by oxygen tension [20], acts as an essential catabolic regulator of osteoarthritis (OA) by modulating the expression of various catabolic factors, including MMPs, in chondrocytes [21]. HIF-2 $\alpha$ additionally regulates inflammatory RA by modulating angiogenesis, various functions of FLS, and interleukin (IL)6-dependent T-helper $\left(T_{H}\right) 17$ cell differentiation [7]. We initially demonstrated that HIF- $2 \alpha$ modulates the expression of various chemokines in both chondrocytes and FLS. Since no links have been established between HIF-2 $\alpha$ induced chemokines, pannus formation, and cartilage erosion, we focused on the potential role of chondrocytederived chemokines in FLS migration and invasion during RA pathogenesis. Here, we examined the hypothesis that HIF-2 $\alpha$-induced chemokine production regulates interactions of chondrocytes with FLS, resulting in formation and invasion of pannus into cartilage tissue and consequently cartilage erosion in RA-affected joint tissues.

\section{Methods}

\section{Mice and experimental arthritis}

Male DBA/1 J, C57BL/6, Epas1 ${ }^{+/-}$, Epas1 $1^{\mathrm{f} / \mathrm{fl}}$, TNF $\alpha$ transgenic (TG), and $\mathrm{K} / \mathrm{BxN}$ mice were used for experimental RA studies. Epas1 ${ }^{+/}$C57BL/6 mice were backcrossed against the DBA/1 J strain for eight generations to generate Epas $^{+/-}$DBA/1 J [7]. TNF $\alpha$ TG and K/BxN mice were obtained from Taconic Farms (Hudson, NY, USA) and Jackson Laboratory (Bar Harbor, ME, USA), respectively. Mice were housed in specific pathogen-free barrier facilities and used in accordance with protocols approved by the Animal Care and Ethics Committees of Chonnam National University and Gwangju Institute of Science and Technology,
Gwangju, Republic of Korea. Collagen-induced arthritis (CIA), K/BxN serum transfer, and TNF $\alpha$ TG mice were employed as experimental RA models. CIA was induced in DBA/1 J mice using a previously described standard protocol [7]. For $\mathrm{K} / \mathrm{BxN}$ serum transfer, $\mathrm{K} / \mathrm{BxN}$ and control sera were collected from arthritic $\mathrm{K} / \mathrm{BxN}$ and nonarthritic $\mathrm{BxN}$ mice, respectively. $\mathrm{K} / \mathrm{BxN}$ or control serum $(200 \mu \mathrm{l})$ was administered intraperitoneally on days 0 and 2 , and mice sacrificed 14 days after serum transfer [22]. TNF $\alpha$ TG mice (20 weeks old, heterozygous) were used as a model for spontaneous inflammatory arthritis, with nonTG wild-type (WT) littermates as controls [23].

\section{Histological analysis of joint tissue and immunostaining}

Mouse joint tissues were decalcified with $0.5 \mathrm{M}$ ethylenediamine tetraacetic acid (EDTA, pH 8.0) for 2 weeks, embedded in paraffin, and sectioned at $5 \mu \mathrm{m}$ thickness. Synovitis was evaluated via hematoxylin and eosin staining of joint sections, and synovial inflammation (grade 0-4) scored as described previously [7]. The pannus in joint tissues adjacent to cartilage and bone was visualized via hematoxylin/safranin-O staining, and pannus formation scored (grades $0-4$ ) as described previously [7]. Cartilage destruction was visualized and scored using Mankin's method [7]. HIF-2 $\alpha$ in joint sections was immunostained with rabbit anti-HIF- $2 \alpha$ (Santa Cruz Biotechnology, Dallas, TX, USA) antibody. For double immunofluorescence labeling in joint sections, rabbit anti-HIF- $2 \alpha$ (Novus Biologicals, Littleton, CO, USA), rabbit anti-CXCL1 (Abcam, Cambridge, UK), goat anti-CXCL2, and goat anti-CCL5 (R\&D Systems, Minneapolis, MN, USA) primary antibodies were used, followed by incubation with Alexa Fluor 594 goat anti-rabbit IgG or Alexa Fluor 488 goat anti-mouse IgG-conjugated secondary antibody (Thermo Fisher Scientific, Waltham, MA, USA).

\section{FLS and articular chondrocyte culture and conditioned medium preparation}

FLS were isolated from joint synovium of WT and Epas $^{+/-}$mice according to previously described protocols [7]. FLS between passages 4 and 8 were used for further analysis. Flow cytometry using antibodies against the fibroblast marker, CD90, and the macrophage marker, CD14 (Abcam), led to the identification of pure FLS $\left(>90 \% \mathrm{CD}^{+} 0^{+} /<1 \% \mathrm{CD} 14^{+}\right)$. Chondrocytes were isolated from cartilage tissue by digestion with $0.2 \%$ collagenase type II, as described previously $[21,24]$, and cells on culture day 3 used for further treatments. To prepare conditioned medium $(\mathrm{CM})$, chondrocytes or FLS were treated with IL-1 $\beta$ or infected with 800 multiplicity of infection (MOI) of empty adenovirus (Ad-C) or adenovirus expressing HIF- $2 \alpha$ (Ad-Epas1) for 24 hours in the absence of serum. 
RNA isolation, RT-PCR, quantitative RT-PCR, and western blotting

Total RNA was extracted from mouse articular chondrocytes and knee joint cartilage using TRI reagent. For isolation of RNA from knee joints, cartilage tissues were sliced using a surgical blade and RNA isolated using the Purelink RNA mini kit (Thermo Fisher Scientific). RNA was reversetranscribed and cDNA amplified using PCR. Transcript levels were quantified with quantitative real time-PCR (qRT-PCR) performed using SYBR Premix Ex $\mathrm{Taq}^{\mathrm{Tm}}$ (TaKaRa Bio Inc., Shiga, Japan). All qRT-PCR reactions were performed in duplicate, and threshold cycle values from target genes normalized to that of glyceraldehyde-3phosphate dehydrogenase (GAPDH). Primer sequences for PCR were described in an earlier report [7]. Western blot analysis was performed to detect secreted CXCL1, CXCL2, and CCL5 in CM using the following antibodies: rabbit anti-CXCL1 (Abcam), goat anti-CXCL2 (R\&D Systems), and goat anti-CCL5 (R\&D Systems).

\section{Chromatin immunoprecipitation assay}

Chromatin immunoprecipitation (ChIP) assays of mouse articular chondrocytes infected with Ad-Epas1 (800 MOI) were performed using a Magna ChIP ${ }^{\mathrm{rm}}$ kit (EMD Millipore, Billerica, MA, USA) [25]. Primers for the ChIP assay were designed to amplify HIF responsive element (HRE)-containing promoter regions of genes of the indicated chemokines. Sequences of primers for the ChIP assay were as follows: Cxcl1 \#1-F, 5'-CAGATGAGAAACATACTTGAGG-3' and \#1-R, 5'-GGAGAACTGGAGCTATCATG-3'; Cxcl1 \#2-F, 5'-AGCATTCTAAAATAAA CAGGG-3' and \#2-R, 5' -GGCAGATTAACGCATTCTT3'; Cxcl1 \#3-F, 5'-TGGGATAAGAGAGGGTAGATG-3' and \#3-R, 5'-GACGTGCTTCGCTGGAC-3'; Cxcl2-F, 5' CCAAACTGTTAGGTCTCCAC-3' and Cxcl2-R, 5' -CT GAGTGGGTTGGGGAC-3'; Cxcl5 \#1-F, 5'-CCAACC CACTCAGCTTAGG-3' and \#1-R, 5'-GGCGCTAGG CTGAAGTG-3'; Cxcl5 \#2-F, 5'-TGTGTATGTCTGCT TATCTGTCT-3' and \#2-R, 5'-ACACTATTGCTGACA CCTGG-3'; Cxcl10 \#1-F, 5'-CTGAACCAAGGATCTGC TC-3' and \#1-R, 5'-TGTACAAGTTCTCAGTCAAGAT G-3'; Cxcl10 \#2-F, 5' -CTTCCGGCTTCTGTTCTG-3' and \#2-R, 5' -CTAAGTCAGGTTCTAACCATGG-3'; and Ccl5F, 5'-CCAATTTCAGACCCTACC-3' and Ccl5-R, 5'-GG CTCAGAACACATTGCA-3'.

\section{Cell migration and invasion assay}

For the transwell migration and invasion assay, FLS from WT $\left(\right.$ Epas $\left.^{+/+}\right)$or Epas1 ${ }^{+/-}$mice (C57BL/6) were seeded on membranes of inserts with $8.0 \mu \mathrm{m}$ pores (Corning Costar, Corning, NY, USA ) and the lower chambers filled with CM, which served as the chemoattractant. Serum-free CM was prepared from primary cultures of chondrocytes or FLS isolated from WT or Epas1 $1^{+-}$mice (C57BL/6).
For neutralization of chemokines, $0.5 \mu \mathrm{g}$ anti-IgG (control), anti-CXCL2, or anti-CCL5 (R\&D Systems) were added to the lower chamber. For the invasion assay, inserts of the transwell were precoated with matrigel (BD Bioscience, San Jose, CA, USA). After 24 hours of incubation, transwell inserts were fixed with $4 \%$ paraformaldehyde for 10 minutes and stained with $0.05 \%$ crystal violet. Cells on the bottom layer were captured in five randomly selected fields at 200× magnification using a light microscope and quantified with Image J software (NIH, shareware) .

\section{Microarray analysis}

Mouse articular chondrocytes and FLS were infected with Ad-Epas1 or empty virus at an MOI of 800 for 24 hours. Total RNA was extracted from mouse articular chondrocytes or mouse FLS cells using TRI reagent (Molecular Research Center, Cincinnati, OH, USA). Three replicates for each cell type were isolated and processed. RNA of mouse articular chondrocytes was analyzed using Agilent microarrays (Agilent Mouse Whole Genome $4 \times 44 \mathrm{~K}$ Microarray), in accordance with the Agilent protocol (Genomic Tree Inc., Daejeon, Korea ). RNA of mouse FLS was analyzed using Affymetrix GeneChip arrays (Affymatrix GeneChip Mouse Gene 2.0 ST Array) using the Affymetrix protocol (DNALINK Inc., Seoul, Korea). All microarray raw data are available through the GEO database [GEO:GSE73658, GEO:GSE73659].

\section{Statistical analysis}

The nonparametric Mann-Whitney $U$ test was used for analysis of data based on an ordinal grading system, such as synovitis, pannus, and Mankin scores. Data obtained with qRT-PCR assays, cell migration and invasion, and HIF- $2 \alpha-$ positive cells were initially tested for conformation to normal distribution using the Shapiro-Wilk test and subsequently analyzed with Student's $t$ test (pair-wise comparisons) or analysis of variance (ANOVA) with post hoc tests (multicomparison), as appropriate. Significance was accepted at the 0.05 level of probability $(P<0.05)$.

\section{Results}

HIF-2 $a$ is upregulated in both cartilage and pannus of the experimental mouse RA joint

To elucidate the possible links and regulatory mechanisms between pannus formation and cartilage erosion, we initially examined HIF- $2 \alpha$ expression levels in chondrocytes from cartilage and FLS in pannus of inflamed joint tissues. CIA [7], K/BxN serum transfer [22], and TNF $\alpha$ TG mice [23] were employed as inflammatory arthritis models. CIA mice displayed severe synovitis, pannus formation, and cartilage erosion in the ankle joint, along with a marked increase in HIF- $2 \alpha$ protein levels in chondrocytes of damaged cartilage and FLS in pannus adjacent to cartilage tissue (Fig. 1a). Similarly, TNF $\alpha$ TG mice (20 weeks old) exhibited synovitis, 


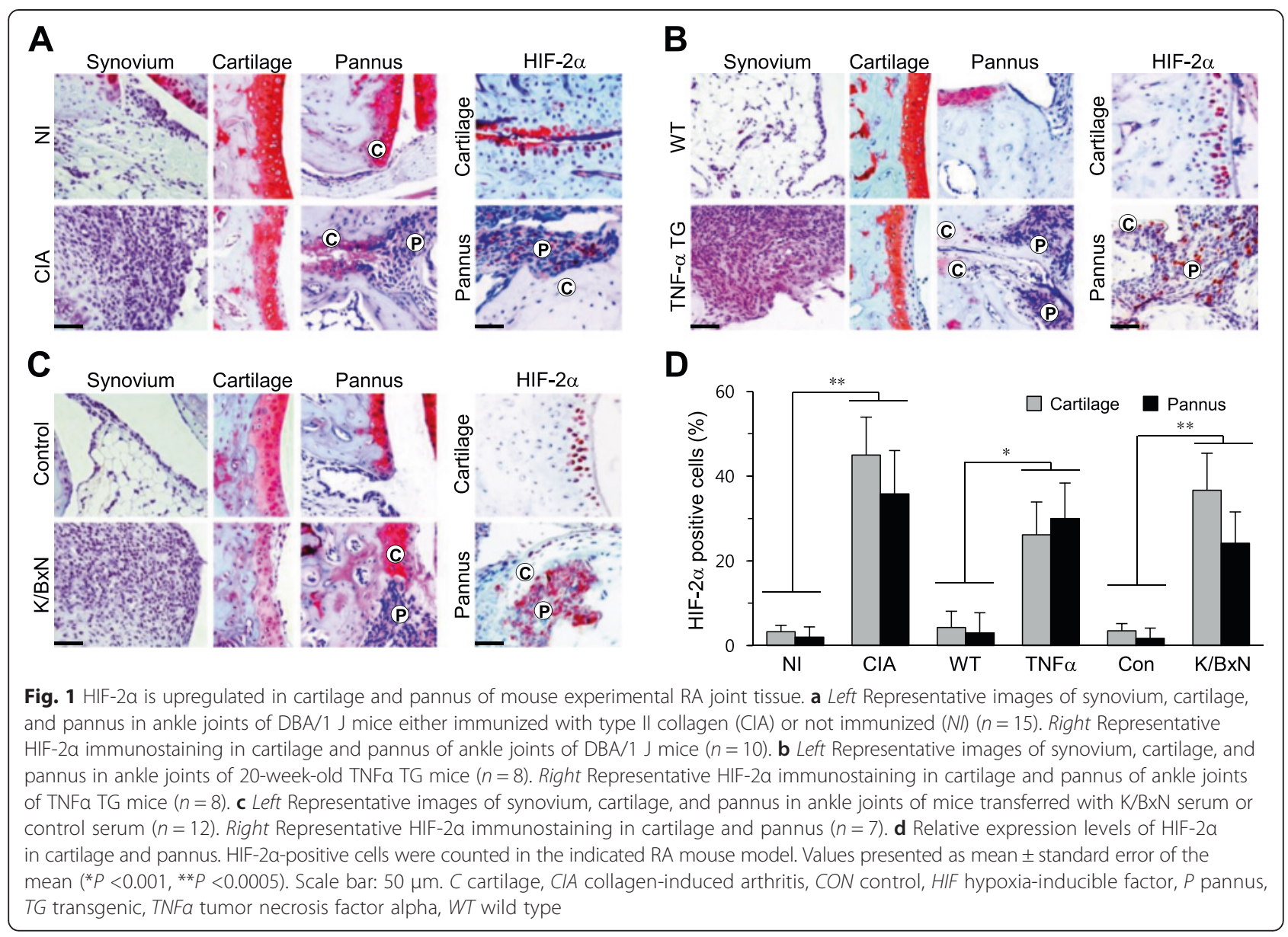

pannus formation, and cartilage erosion with increased HIF$2 \alpha$ levels in chondrocytes and FLS of cartilage and pannus, respectively (Fig. 1b). Additionally, HIF-2 $\alpha$ was markedly upregulated in cartilage and pannus in the $\mathrm{K} / \mathrm{BxN}$ serum transfer model, with severe synovitis and cartilage erosion (Fig. 1c). Quantitation of HIF-2 $\alpha$-positive cells in cartilage and pannus of RA-affected joints revealed significantly increased numbers of chondrocytes and FLS in all the models of inflammatory arthritis examined (Fig. 1d).

\section{HIF-2a upregulates chemokine expression in both FLS and chondrocytes}

Next, we examined whether HIF-2 $\alpha$ regulates chemokine expression in chondrocytes and FLS. Microarray analyses of HIF-2 $\alpha$-overexpressing FLS revealed markedly increased mRNA levels of CXCL3 (22.1-fold), CXCL2 (9.3-fold), CCL20 (3.7-fold), CXCL1 (3.5-fold), and CXCL5 (1.6-fold) (Fig. 2a). HIF-2 $\alpha$ overexpression in chondrocytes also led to a marked increase in the mRNA levels of CXCL2 (37.0-fold), CXCL5 (23.0-fold), CXCL1 (21.9-fold), CCL5 (15.3-fold), CXCL10 (15.2-fold), CCL7 (9.4-fold), CCL2 (7.6-fold), CXCL7 (5.1-fold), CCL8 (4.2-fold), and CXCL11 (3.7-fold) (Fig. 2b). Upregulation of chemokines by HIF$2 \alpha$ in primary culture chondrocytes was further verified with RT-PCR and qRT-PCR (Fig. 2c, d). Using the ChIP assay, we additionally investigated whether these chemokines are direct target genes of HIF- $2 \alpha$ in mouse chondrocytes. Promoters of Cxcl1, Cxcl2, Cxcl5, Cxcl10, Ccl2, and $\mathrm{Ccl} 5$, but not $\mathrm{Ccl} 2$ and $\mathrm{Ccl} 7$, contain one or more hypoxia response element (HRE) motifs (-(A/G)CGTG-) (Fig. 2e). ChIP data revealed direct binding of HIF- $2 \alpha$ to promoters of $C x c l 1, C x c l 2, C x c l 5, C x c l 10$, and $C c l 5$ in primary culture chondrocytes (Fig. 2f).

Next, we examined whether HIF- $2 \alpha$ regulates the expression of chemokines in mouse cartilage tissue. Consistent with previous findings [7], intra-articular (IA) injection of Ad-Epas1 promoted pannus formation (Fig. 3a). RT-PCR and qRT-PCR analyses of cartilage tissue revealed that HIF- $2 \alpha$ induces a significant increase in mRNA levels of the chemokines examined (Fig. 3b). Furthermore, HIF-2 $\alpha$-overexpressing chondrocytes in cartilage tissue of mice IA injected with Ad-Epas1 showed increased levels of CXCL1, CXCL2, and CCL5 proteins, which co-localized with HIF-2 $\alpha$ (Fig. 3c). 


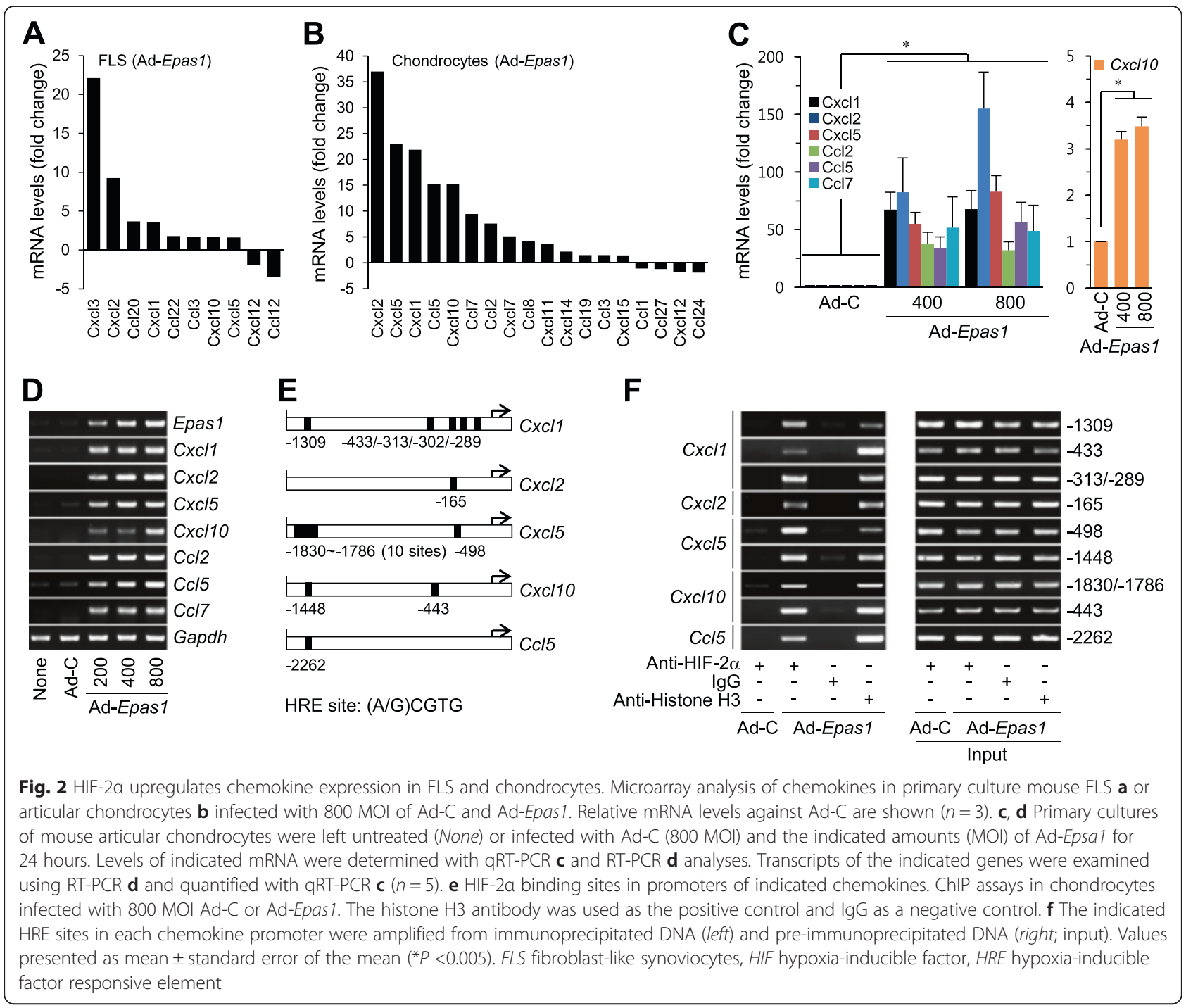

Similarly, CXCL1, CXCL2, and CCL5 proteins were increased in HIF-2 $\alpha$-overexpressing chondrocytes in cartilage tissue of CIA mice and co-localized with HIF- $2 \alpha$ (Fig. 3d). Our results collectively indicate that HIF- $2 \alpha$ upregulates chemokines in both primary culture chondrocytes and cartilage tissue.

IL-1 $\beta$ is one of the major proinflammatory cytokines regulating RA pathogenesis [26]. IL-1 $\beta$ also transcriptionally upregulates HIF- $2 \alpha$ in FLS and chondrocytes $[7,21]$. Accordingly, we examined whether IL-1 $\beta$ stimulates chemokine expression via the HIF- $2 \alpha$ pathway in chondrocytes. IL-1 $\beta$ enhanced chemokine expression in WT chondrocytes (Fig. 4a, b). This upregulation was markedly inhibited in Epas1 ${ }^{+-}$chondrocytes (Fig. 4c). We previously showed that deletion of one allele of Epas1 significantly inhibits the hallmarks of RA, including synovitis, cartilage erosion, and pannus formation [7]. Consistently, Epas1 ${ }^{+-}$DBA/1 J mice under CIA conditions showed marked reduction of CXCL1, CXCL2, and CCL5 expression, compared with their corresponding WT littermates (Fig. 4d). These findings suggest that regulation of chemokines in RA cartilage is controlled by HIF- $2 \alpha$.

\section{HIF-2a-induced chemokines by chondrocytes regulate FLS} migration and invasion

FLS, a major cell type in pannus tissue, migrate, attach to, invade, and destroy cartilage tissue by secreting proteases within the RA joints [4]. Since a major function of chemokines is recruitment of adjacent cells as a chemoattractant, we used an in vitro modified Boyden chamber assay to further examine whether increased expression of chemokines by HIF- $2 \alpha$ in FLS and chondrocytes plays a role in FLS migration and invasion. Our data initially confirmed that CM from FLS infected with Ad-Epas1 stimulates both migration and invasion of FLS (Fig. 5a). 


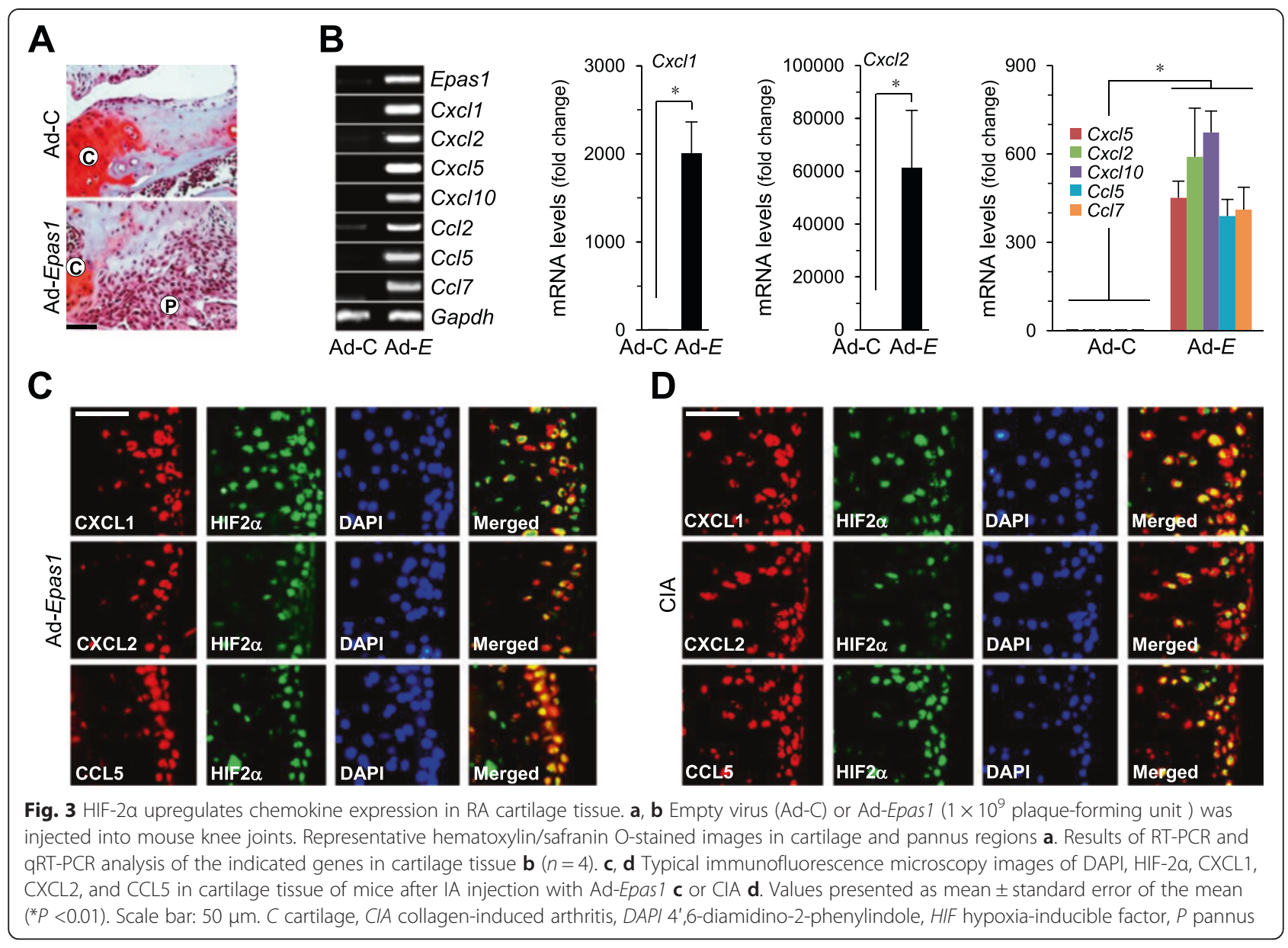

Next, we examined whether HIF-2 $\alpha$-induced chemokines by chondrocytes also regulate migration and invasion of FLS. Notably, CM from Ad-Epas1-infected chondrocytes promoted both migration and invasion of FLS (Fig. 5b). The correlation between chondrocyteoriginating HIF- $2 \alpha$ and chemokine function in FLS migration and invasion was further validated. CM from chondrocytes treated with IL-1 $\beta$ stimulated the migration and invasion of WT $\left(\right.$ Epas $\left.^{+/+}\right)$FLS (Fig. 5c). However, Epas1 ${ }^{+/-}$FLS exhibited significantly reduced migration and invasion in the presence of IL-1 $\beta$-treated chondrocyte CM (Fig. 5c). Furthermore, addition of anti-CXCL2 and anti-CCL5 antibodies to CM of IL-1 $\beta$ treated chondrocytes blocked FLS migration (Fig. 5d). Based on the results, we propose that HIF- $2 \alpha$-induced chemokines by chondrocytes regulate FLS migration and invasion.

\section{Cartilage-specific knockdown of HIF-2a inhibits FLS migration at the cartilage-pannus interface}

Finally, we elucidated the in vivo significance of HIF-2 $\alpha$ derived chemokine production in chondrocytes during RA cartilage destruction. For this purpose, HIF- $2 \alpha$ was locally downregulated in joint tissues via IA injection of Ad-Cre in Epas1 ${ }^{\mathrm{fl} / \mathrm{fl}}$ mice and experimental RA induced by $\mathrm{K} / \mathrm{BxN}$ serum transfer. Immunostaining in synovium and cartilage revealed that Ad-Cre injection effectively reduced the levels of HIF- $2 \alpha$ induced by $\mathrm{K} / \mathrm{BxN}$ serum transfer (Fig. 6a). Local deletion of Epas1 in joint tissues significantly suppressed all the hallmarks of RA, including synovitis and synovial hyperplasia, pannus formation and invasion into calcified cartilage and bone, and cartilage destruction (Fig. 6b, c). To elucidate the role of HIF-2 $\alpha$-regulated chemokines in cartilage, we used chondrocyte-specific conditional knockout (cKO) mice (Epas $1^{\mathrm{fl} / \mathrm{fl}} ;$ Col2a1-cre) in a $\mathrm{K} / \mathrm{BxN}$ serum transfer model. In $\mathrm{cKO}$ mice, $\mathrm{K} / \mathrm{BxN}$ serum transfer did not cause upregulation of HIF- $2 \alpha$ in cartilage whereas HIF- $2 \alpha$ was upregulated in synovial tissue (Fig. 6d). Consistently, Epas $1^{\mathrm{fl} / \mathrm{fl}} ;$ Col2a1-cre mice exhibited significantly reduced cartilage erosion and pannus formation adjacent to cartilage tissue after $\mathrm{K} / \mathrm{BxN}$ serum transfer, compared with Epas $^{\mathrm{fl} / \mathrm{fl}}$ mice (Fig. 6e, f). However, K/BxN serum transfer caused a similar degree of synovitis and bone loss at the bone-pannus junction (Fig. 6e, f), indicating that HIF- $2 \alpha$ knockout in cartilage inhibits pannus expansion 


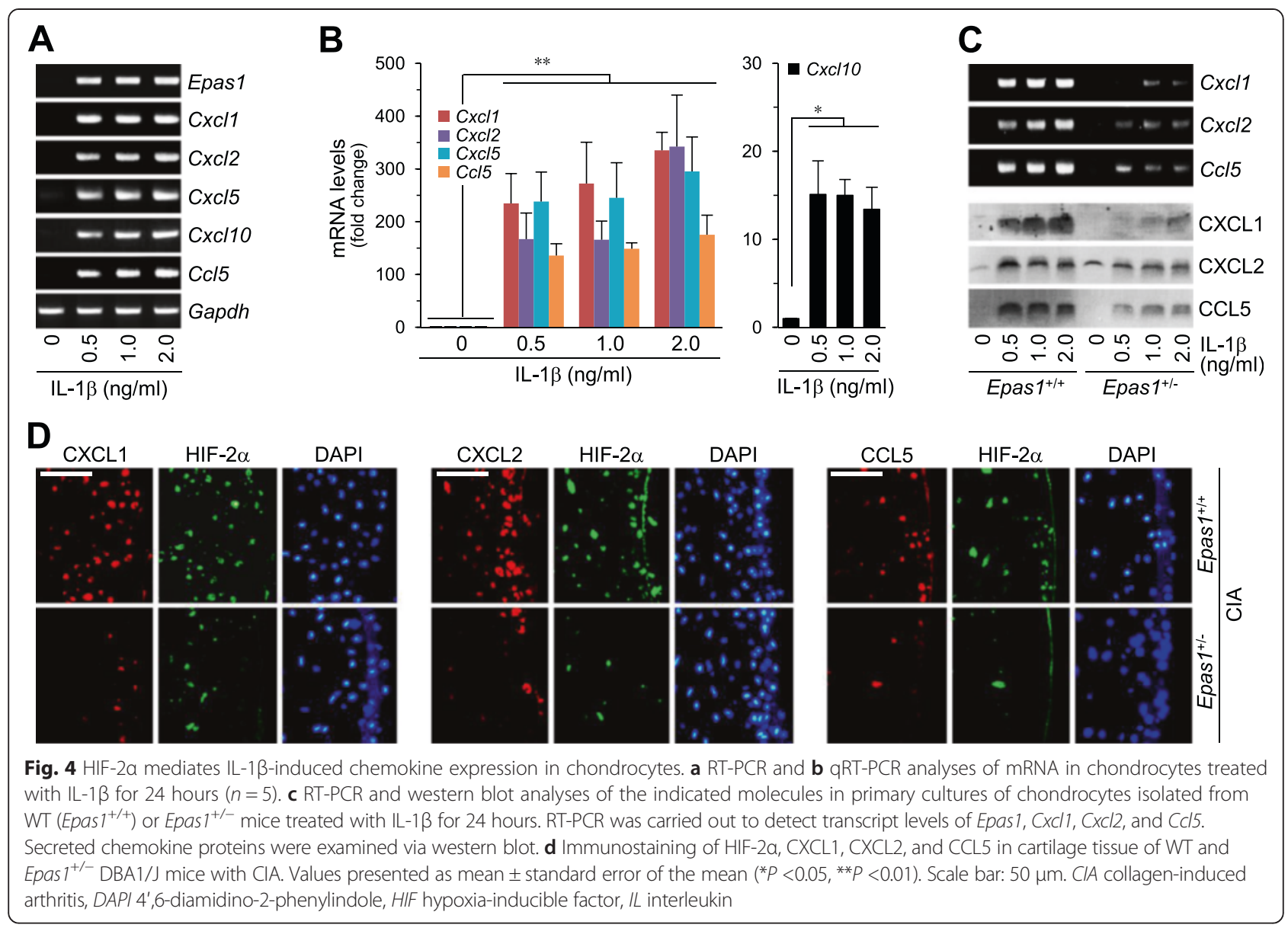

and FLS migration in RA. Our collective findings suggest that HIF-2 $\alpha$-stimulated chemokines in chondrocytes regulate FLS motility, resulting in pannus invasion into the cartilage during RA pathogenesis.

\section{Discussion}

Our current results suggest that RA-triggering factors, such as IL-1 $\beta$ and HIF-2 $\alpha$, enhance the expression of several chemokines in chondrocytes, including Cxcl1, Cxcl2, Cxcl5, Cxcl10, Ccl2, Ccl5, and Ccl7. These HIF$2 \alpha$-induced chemokines in chondrocytes stimulate migration and invasion of FLS, leading to pannus expansion adjacent to cartilage tissue of RA joints. Functional blockage of HIF-2 $\alpha$-induced chemokines, such as $C x c l 2$ and $\mathrm{Ccl}$, ameliorated FLS motility. Moreover, Cxcl1, Cxcl2, Cxcl5, Cxcl10, and Ccl5 were identified as direct target genes of HIF- $2 \alpha$ in chondrocytes. Local deletion of HIF- $2 \alpha$ in joint tissues or conditional knockout in cartilage tissue inhibited pannus formation adjacent to cartilage tissue and cartilage destruction caused by $\mathrm{K} / \mathrm{BxN}$ serum transfer, demonstrating the significance of chondrocyte-originated chemokines in pannus expansion and cartilage destruction during RA pathogenesis.
Formation of invasive pannus, a thickened layer of synovial tissue that erodes cartilage and bone, is the pathologic hallmark of RA [2, 3]. FLS is a main effector cell type in the progression of RA, promoting pannus formation with cartilage erosion and progressive joint destruction by secreting diverse cytokines, chemokines, and MMPs $[5,8,9]$. Here, we demonstrated upregulation of HIF- $2 \alpha$ in chondrocytes from damaged cartilage and FLS in the pannus region of various RA mouse models, including CIA, TNF $\alpha$ TG, and $\mathrm{K} / \mathrm{BxN}$ serum transfer. To elucidate the potential functions of HIF- $2 \alpha$, we screened the gene expression profiles in HIF-2 $\alpha$ overexpressing chondrocytes and FLS via microarray analysis. Functional enrichment analysis of microarray data revealed upregulation of genes associated with RA pathogenesis, such as those involved in extracellular matrix (ECM) remodeling and inflammatory responserelated, cell migration-related and invasion-related processes (data not shown). Among these, we focused on chemokines expressed in both chondrocytes and FLS. Chemokines regulate chemotaxis in nearby responsive cells and are implicated in chronic inflammatory diseases, such as RA, atherosclerosis, and adipose inflammation [14]. 




Release of chemoattractants by FLS signals various immune cells to migrate into the RA synovium [4]. Chemokines, such as $\mathrm{CX}_{3} \mathrm{CL} 1, \mathrm{CCL} 2$, and CXCL10, and their receptors, CCR2, CCR5, and CXCR3, regulate FLS migration or invasion during RA pathogenesis $[16,27,28]$. In addition to FLS, chondrocytes produce various chemokines and related catabolic factors during cartilage destruction. For instance, human OA chondrocytes generate various chemokines (CXCL1, CCL2, and CCL5) and chemokine receptors (CCR1, CCR2, CCR3, CCR5, CXCR1, and CXCR2) by proinflammatory cytokines [17-19]. CXCR1/CXCR2 production by human OA chondrocytes stimulates MMP3 release [19], and constitutively expressed CXCR2 in normal chondrocytes maintains cartilage homeostasis [29]. However, the functions and regulatory mechanisms of HIF- $2 \alpha$-induced chemokines in RA pathogenesis are yet to be elucidated. Our current results clearly demonstrate that HIF- $2 \alpha$ upregulates various chemokines as direct target genes in primary culture chondrocytes. Additionally, upregulated HIF- $2 \alpha$ in cartilage tissue stimulates chemokine expression in chondrocytes. We detected three CCL (CCL2, CCL5, and CCL7) and four CXCL chemokine families (CXCL1, CXCL2, CCXCL5, and CXCL10) that were highly upregulated by IL-1 $\beta$ or HIF-2 $\alpha$. Among these, Cxcl1, Cxcl2, Cxcl5, $\mathrm{Cxcl10}$, and $\mathrm{Ccl} 5$, but not $\mathrm{Ccl} 2$ and $\mathrm{Ccl} 7$, were identified as direct target genes of HIF-2 $\alpha$. Ccl2 and $\mathrm{Ccl}$ genes may be regulated indirectly or independently of HIF- $2 \alpha$. NF- $-\mathrm{B}$ is a potential transcription factor responsible, since the promoter region contains a NF- $\mathrm{kB}$ binding site and IL-1 $\beta$ induced HIF- $2 \alpha$ upregulation is controlled by NF- $\mathrm{kB}$ signaling [21]. Furthermore, our data show that HIF-2 $\alpha-$ induced chemokines by chondrocytes stimulate in vitro migration and invasion of FLS. The observation that addition of blocking antibodies against CXCL2 and CCL5 to $\mathrm{CM}$ of chondrocytes abrogates FLS migration clearly demonstrates an essential role of HIF- $2 \alpha$-induced chemokines by chondrocytes in FLS motility. Although chemokines are produced by both chondrocytes and FLS, it remains to be established whether HIF- $\alpha$-induced chemokines by chondrocytes act in a manner complementary to or redundant with those produced by FLS in the development and pathogenesis of experimental RA. Different chemokine subtypes are upregulated in FLS and 


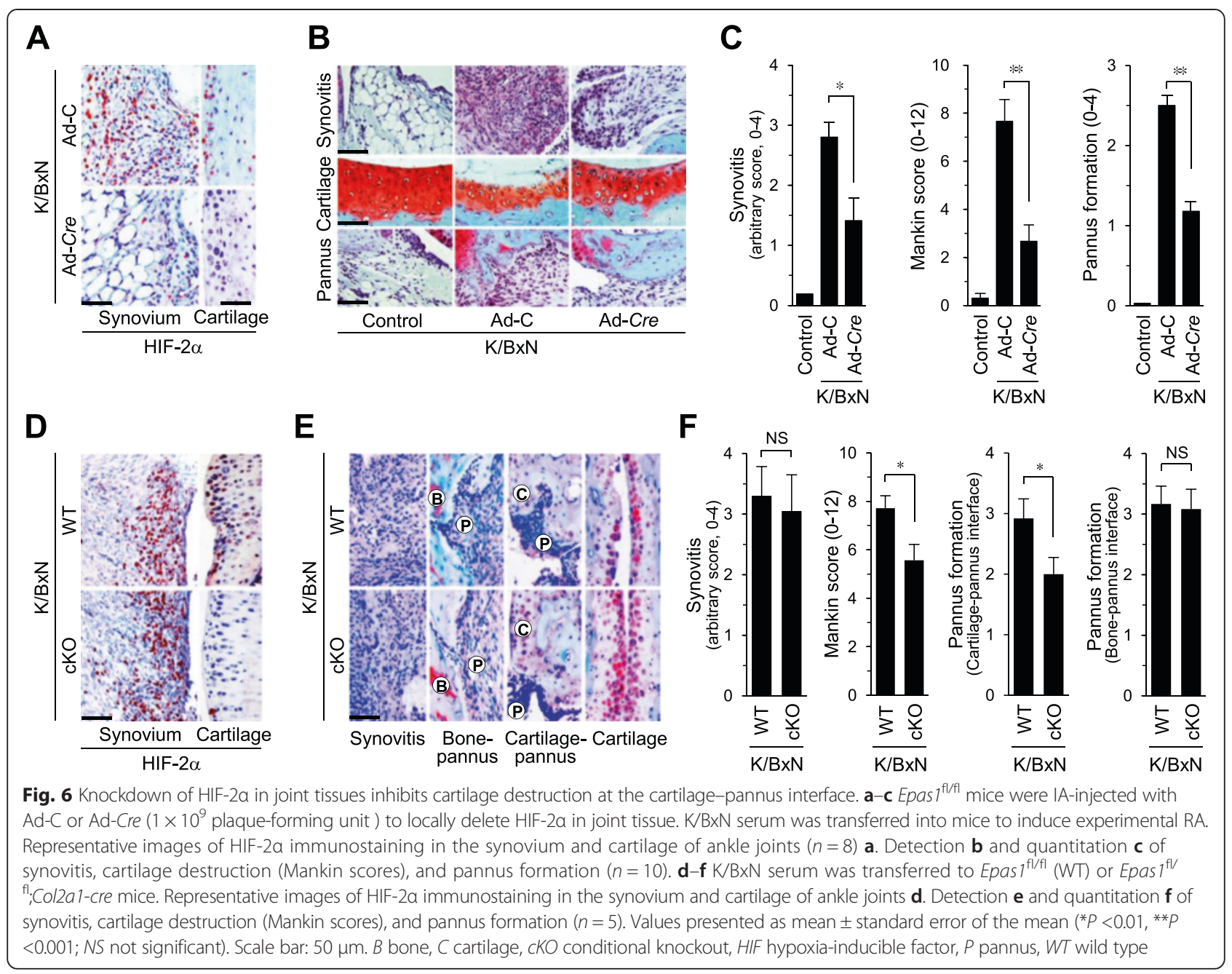

chondrocytes. For instance, CXCL3 and CXCL2 are the most highly upregulated in Ad-Epas1-infected FLS, while CXCL2, CXCL5, CXCL1, and CCL5 are significantly upregulated by HIF-2 $\alpha$-overexpressing chondrocytes. Among these, CXCL2 expression was the most upregulated in AdEpas1-infected chondrocytes. Chondrocyte-derived CXCL2 appears to be a major chemoattractant triggering FLS migration, based on data showing that blockage of CXCL2 with specific antibodies inhibits FLS migration in IL-1 $\beta$ treated CM. All chemokines exert biological effects by stimulating G protein-linked receptors [14]. Microarray analysis revealed the presence of CXCR2 (for CXCL1, CXCL2, and CXCL5), CXCR3 (for CXCL10), and CCR5 (for CCL5) [14] in both mouse FLS and articular chondrocytes, although the expression levels of these receptors were not affected by HIF- $2 \alpha$ overexpression (data not shown).

Chondrocytes in cartilage tissue are embedded in cartilage matrix, raising the question of how chondrocytederived chemokines regulate the motility of FLS, which are localized in synovial tissues. One possibility is that chemokines diffuse from the cartilage matrix to affect synovial cells [30]. Although we did not validate this theory in the current study, we were able to demonstrate the significance of chondrocyte-derived chemokines in the expansion of invasive pannus in vivo. Local deletion of HIF$2 \alpha$ in joint tissues via IA injection of Ad-Cre in Epast ${ }^{\mathrm{t} / \mathrm{fl}}$ mice suppressed pannus formation and cartilage destruction. Furthermore, cKO of HIF- $2 \alpha$ in cartilage tissue, which inhibited chemokine production, led to sufficient blockade of pannus formation adjacent to cartilage tissue, but not in bone tissue, resulting in inhibition of cartilage destruction. Cartilage is eroded by breakdown of ECM via the action of several proteases, such as MMPs and cathepsin, during RA. Our group previously showed that stimulation of chondrocytes with IL-1 $\beta$ increases secretion of MMPs in association with marked HIF-2 $\alpha$ transcriptional activity [7, 21]. MMPs participate in ECM remodeling and play important roles in the progressive destruction of joints in RA. FLS are considered a major effector cell type involved in cartilage erosion, based on their ability to produce massive 
amounts of degradative enzymes, such as MMPs. Since both FLS and cartilage are major cell types that produce ECMdegrading enzymes $[7,21]$, cartilage erosion during RA may be attributable to a multistep and complex process including "FLS attachment to cartilage" and "synthesis of enzymes from FLS and cartilage that degrade the ECM". This theory is supported by the finding that deletion of HIF-2 $\alpha$ in both synovium and cartilage exerts a greater ameliorating effect on cartilage destruction than local deletion in cartilage.

\section{Conclusions}

In summary, HIF- $2 \alpha$ is overexpressed in damaged chondrocytes as well as FLS in the pannus region in several experimental RA mouse models, and directly regulates the expression of Cxcl1, Cxcl2, Cxcl5, Cxcl10, and Ccl5. In addition, we have identified a previously unrecognized role of chondrocyte-derived chemokines and provided a mechanistic explanation for the regulatory processes underlying the invasive properties of FLS. Although our findings from experimental RA mouse models remain to be verified in human RA pathogenesis, we carefully suggest new insights into the effects of chondrocytes on synovial cell motility into the cartilage-pannus interface in RA pathogenesis and highlight the relevance of HIF- $2 \alpha$ as a potential novel therapeutic target in RA.

\section{Abbreviations \\ ANOVA: Analysis of variance; ChIP: Chromatin immunoprecipitation; CIA: Collagen-induced arthritis; CKO: Conditional knockout; CM: Conditioned medium; ECM: Extracellular matrix; EDTA: Ethylenediamine tetraacetic acid; FLS: Fibroblast-like synoviocytes; GAPDH: Glyceraldehyde-3-phosphate dehydrogenase; HIF: Hypoxia-inducible factor; HRE: Hypoxia-inducible factor responsive element; IA: Intra-articular; IL: Interleukin; MMP: Matrix metalloproteinase; MOI: Multiplicity of infection; OA: Osteoarthritis; qRT-PCR: Quantitative RT-PCR; RA: Rheumatoid arthritis; TG: Transgenic; $T_{H}:$ T-helper ; TNFa: Tumor necrosis factor alpha; WT: Wild type.}

\section{Competing interests}

The authors declare that they have no competing interests.

\begin{abstract}
Authors' contributions
$\mathrm{YHH}$ and $\mathrm{GL}$ designed and performed most in vitro and in vivo studies. $\mathrm{YHH}$ conceived the project, performed histological evaluations, and drafted the manuscript. GL carried out the immunoassays, performed the statistical analysis, and revised the manuscript. K-BL participated in the design of the study and helped to draft the manuscript. J-TK participated in the animal study, analyzed the data, and helped to draft the manuscript. J-SC and J-HR conceived the project, drafted the manuscript, and was responsible for the overall design and oversight of the project. All authors read and approved the final version of the manuscript.
\end{abstract}

\section{Acknowledgements}

This work was supported by grants from the National Research Foundation of Korea (2011-0030121), the Korea Health Technology R\&D Project through the Korea Health Industry Development Institute, funded by the Ministry of Health \& Welfare (H112C1606 and HI14C-3484-080014), and Chonnam National University Hospital Biomedical Research Institute (CRI 13045-22).

\section{Author details}

'Biolmaging and Cell Dynamics Research Center, Gwangju Institute of Science and Technology, 123 Cheomdangwagi-ro, Buk-gu, Gwangju 61005, Republic of Korea. ${ }^{2}$ Cell Dynamics Research Center and School of Life Science, Gwangju Institute of Science and Technology, 123
Cheomdangwagi-ro, Buk-gu, Gwangju 61005, Republic of Korea. ${ }^{3}$ Department of Orthopedic Surgery, Chonnam National University Medical School and Hospital, 42 Jebong-ro, Dong-gu, Gwangju 61469, Republic of Korea. ${ }^{4}$ Department of Pharmacology and Dental Therapeutics, School of Dentistry, Chonnam National University, 77 Yongbong-ro, Buk-gu, Gwangju 61186, Republic of Korea. ${ }^{5}$ Research Center for Biomineralization Disorders, School of Dentistry, Chonnam National University, 77 Yongbong-ro, Buk-gu, Gwangju 61186, Republic of Korea.

Received: 18 June 2015 Accepted: 9 October 2015

Published online: 29 October 2015

\section{References}

1. Lefèvre S, Knedla A, Tennie C, Kampmann A, Wunrau C, Dinser R, et al. Synovial fibroblasts spread rheumatoid arthritis to unaffected joints. Nat Med. 2009;15:1414-20.

2. Mclnnes IB, Schett $\mathrm{G}$. The pathogenesis of rheumatoid arthritis. N Engl J Med. 2011;365:2205-19.

3. Goronzy JJ, Weyand CM. Developments in the scientific understanding of rheumatoid arthritis. Arthritis Res Ther. 2009;11:249-62.

4. Bartok B, Firestein GS. Fibroblast-like synoviocytes: key effector cells in rheumatoid arthritis. Immunol Rev. 2010;233:233-55.

5. Bottini N, Firestein GS. Duality of fibroblast-like synoviocytes in RA: passive responders and imprinted aggressors. Nat Rev Rheumatol. 2013;9:24-33.

6. Lefèvre $S$, Meier FMP, Neumann E, Muller-Ladner U. Role of synovial fibroblasts in rheumatoid arthritis. Curr Pharm Des. 2015;21:130-41.

7. Ryu JH, Chae CS, Kwak JS, Oh H, Shin Y, Huh YH, et al. Hypoxia-Inducible Factor-2a is an essential catabolic regulator of inflammatory rheumatoid arthritis. Plos Biol. 2014;12:e1001881.

8. Noss EH, Brenner MB. The role and therapeutic implications of fibroblast-like synoviocytes in inflammation and cartilage erosion in rheumatoid arthritis. Immunol Rev. 2008;223:252-70.

9. Abeles AM, Pillinger MH. The role of the synovial fibroblast in rheumatoid arthritis: cartilage destruction and the regulation of matrix metalloproteinases. Bull NYU Hosp Jt Dis. 2006;64:20-4.

10. Sawai H, Park YW, He X, Goronzy JJ, Weyand CM. Fractalkine mediates T cell-dependent proliferation of synovial fibroblasts in rheumatoid arthritis. Arthritis Rheum. 2007;56:3215-25.

11. Szekanecz Z, Kim J, Koch AE. Chemokines and chemokine receptors in rheumatoid arthritis. Semin Immunol. 2003;15:15-21.

12. Koch AE. Chemokines and their receptors in rheumatoid arthritis: future targets? Arthritis Rheum. 2005;52:710-21.

13. Patel DD, Zachariah JP, Whichard LP. CXCR3 and CCR5 ligands in rheumatoid arthritis synovium. Clin Immunol. 2001;98:39-45.

14. Jin T, Xu X, Hereld D. Chemotaxis, chemokine receptors and human disease. Cytokine. 2008;44:1-8.

15. Charo IF, Ransohoff RM. The many roles of chemokines and chemokine receptors in inflammation. N Engl J Med. 2006;354:610-21.

16. García-Vicuña R, Gómez-Gaviro MV, Domínguez-Luis MJ, Pec MK, González-Alvaro I, Alvaro-Gracia JM, et al. CC and CXC chemokine receptors mediate migration, proliferation, and matrix metalloproteinase production by fibroblast-like synoviocytes from rheumatoid arthritis patients. Arthritis Rheum. 2004;50:3866-77.

17. Pulsatelli L, Dolzani P, Piacentini A, Silvestri T, Ruggeri R, Gualtieri G, et al. Chemokine production by human chondrocytes. J Rheumatol. 1999;26:1992-2001

18. Borzi RM, Mazzetti I, Cattini L, Uguccioni M, Baggiolini M, Facchini A. Human chondrocytes express functional chemokine receptors and release matrix-degrading enzymes in response to C-X-C and C-C chemokines. Arthritis Rheum. 2000;43:1734-41.

19. Kanbe K, Takagishi K, Chen Q. Stimulation of matrix metalloprotease 3 release from human chondrocytes by the interaction of stromal cell-derived factor 1 and CXC chemokine receptor 4. Arthritis Rheum. 2002:46:130-7.

20. Greer SN, Metcalf $J$ L, Wang Y, Ohh M. The updated biology of hypoxia-inducible factor. EMBO J. 2012;31:2448-60.

21. Yang S, Kim J, Ryu JH, Oh H, Chun CH, Kim BJ, et al. Hypoxia-inducible factor-2a is a catabolic regulator of osteoarthritic cartilage destruction. Nat Med. 2010;16:687-93.

22. Matsumoto I, Staub A, Benoist C, Mathis D. Arthritis provoked by linked T and B cell recognition of a glycolytic enzyme. Science. 1999;286:1732-5. 
23. Keffer J, Probert L, Cazlaris H, Georgopoulos S, Kaslaris E, Kioussis D, et al. Transgenic mice expressing human tumour necrosis factor: a predictive genetic model of arthritis. EMBO J. 1991;10:4025-31.

24. Gosset M, Berenbaum F, Thirion S, Jacques C. Primary culture and phenotyping of murine chondrocytes. Nat Protoc. 2008;3:1253-60.

25. Ryu JH, Yang S, Shin Y, Rhee J, Chun CH, Chun JS. Interleukin-6 plays an essential role in hypoxia-inducible factor 2a-induced experimental osteoarthritic cartilage destruction in mice. Arthritis Rheum. 2011;63:2732-43.

26. McInnes IB, Schett G. Cytokines in the pathogenesis of rheumatoid arthritis. Nat Rev Immunol. 2007;7:429-42.

27. Volin MV, Huynh N, Klosowska K, Chong KK, Woods JM. Fractalkine is a novel chemoattractant for rheumatoid arthritis fibroblast-like synoviocyte signaling through MAP kinases and Akt. Arthritis Rheum. 2007:56:2512-22.

28. Laragione T, Brenner M, Sherry B, Gulko PS. CXCL10 and its receptor CXCR3 regulate synovial fibroblast invasion in rheumatoid arthritis. Arthritis Rheum. 2011;63:3274-83

29. Sherwood J, Bertrand J, Nalesso G, Poulet B, Pitsillides A, Brandolini L, et al. A homeostatic function of CXCR2 signalling in articular cartilage. Ann Rheum Dis. 2014;pii: annrheumdis-2014-205546.

30. Pap T, Van Der Laan WH, Aupperle KR, Gay RE, Verheijen JH, Firestein GS, et al. Modulation of fibroblast-mediated cartilage degradation by articular chondrocytes in rheumatoid arthritis. Arthritis Rheum. 2000;43:2531-6.

\section{Submit your next manuscript to BioMed Central and take full advantage of:}

- Convenient online submission

- Thorough peer review

- No space constraints or color figure charges

- Immediate publication on acceptance

- Inclusion in PubMed, CAS, Scopus and Google Scholar

- Research which is freely available for redistribution 